\title{
Free Convection Heat and Mass Transfer MHD Flow in a Vertical Porous Channel in the Presence of Chemical Reaction
}

\author{
R. N. Barik, ${ }^{1}$ G. C. Dash, ${ }^{2}$ and M. Kar ${ }^{3}$ \\ ${ }^{1}$ Department of Mathematics, Trident Academy of Technology, Infocity, Bhubaneswar, Odisha 751024, India \\ ${ }^{2}$ Department of Mathematics, S.O.A. University, Bhubaneswar, Odisha 751030, India \\ ${ }^{3}$ Department of Mathematics, Christ College, Cuttack, Odisha, India \\ Correspondence should be addressed to R. N. Barik; rnbmath22@yahoo.com
}

Received 20 May 2013; Revised 18 September 2013; Accepted 23 September 2013

Academic Editor: Hideki Tsuge

Copyright (C) 2013 R. N. Barik et al. This is an open access article distributed under the Creative Commons Attribution License, which permits unrestricted use, distribution, and reproduction in any medium, provided the original work is properly cited.

\begin{abstract}
The objective of the present study is to examine the fully developed free convective MHD flow of an electrically conducting viscous incompressible fluid in a vertical porous channel under influence of asymmetric wall temperature and concentration in the presence of chemical reaction. The heat and mass transfer coupled with diffusion-thermo effect renders the present analysis interesting and curious. The analytical solution by Laplace transform technique of partial differential equations is used to obtain the expressions for the velocity, temperature, and concentration. It is observed that under the influence of dominating mass diffusivity over thermal diffusivity with stronger Lorentz force the velocity is reduced at all points Further, low rate of thermal diffusion delays the attainment of free stream state. Flow of aqueous solution in the presence of heavier species is prone to back flow.
\end{abstract}

\section{Introduction}

In many transport processes and industrial applications, transfer of heat and mass occurs simultaneously as a result of combined buoyancy effects of thermal diffusion and diffusion of chemical species. Unsteady natural convection of heat and mass transfer is of great importance in designing control systems for modern free convection heat exchangers. More recently, Jha and Ajibade [1] have studied the heat and mass transfer aspect of the flow of a viscous incompressible fluid in a vertical channel considering the Dufour effect.

Soundalgekar and Akolkar [2] studied the effect of mass transfer and free convection currents on the flow past an impulsively started infinite vertical plate and observed that the presence of foreign gasses in the flow domain leads to reduce the shear stress and rate of mass transfer significantly.

In nature, flow occurs due to density differences caused by temperature as well as chemical composition gradients. Therefore, it warrants the simultaneous consideration of temperature difference as well as concentration difference when heat and mass transfer occurs simultaneously. It has been found that an energy flux can be created not only by temperature gradients but by composition gradients also. This is called Dufour effect. If, on the other hand, mass fluxes are created by temperature gradients, it is called the Soret effect.

The Soret and the Dufour effects have been found to be useful as the Soret effect is utilized for isotope separation and, in a mixture of gases of light and medium molecular weight, the Dufour effect is found to be of considerable order of magnitude such that it cannot be neglected. In view of importance of the diffusion-thermo effect Kafoussias and Williams [3] have studied the effects of thermal diffusion and diffusion-thermo on mixed free and forced convective and mass transfer boundary layer flow with temperature dependent viscosity. Jha and Singh [4], Kafoussias [5], and Alam et al. $[6,7]$ have contributed significantly to this field of study. Recently, Beg et al. [8] studied chemically reacting mixed convective heat and mass transfer along inclined and vertical plates considering Soret and Dufour effects. More recently, Dursunkaya and Worek [9] have studied the diffusion-thermo and thermal diffusion effects in transient and steady natural convections from a vertical surface.

MHD flow with thermal diffusion and chemical reaction finds numerous applications in various areas such as thermo nuclear fusion, liquid metal cooling of nuclear reactions, and electromagnetic casting of metals. Abreu et al. [10] have 
studied the boundary layer flows with Dufour and Soret effects. Osalusi et al. [11] have worked on mixed and free convective heat and mass transfer of an electrically conducting fluid considering Dufour and Soret effects. Anghel et al. [12] and Postelnicu [13] obtained numerical solutions of chemical reacting mixed convective heat and mass transfer along inclined and vertical plates with the Soret and the Dufour effects and concluded that skin friction increases with a positive increase in the concentration-to-thermalbuoyancy ratio parameter.

The analysis of natural convection heat and mass transfer near a moving vertical plate has received much attention in recent times due to its wide application in engineering and technological processes. There are applications of interest in which combined heat and mass transfer by natural convection occurs between a moving material and ambient medium, such as the design and operation of chemical processing equipment, design of heat exchangers, transpiration cooling of a surface, chemical vapour deposition of solid layer, nuclear reactor, and many manufacturing processes like hot rolling, hot extrusion, wire drawing, continuous casting, and fiber drawing. Gebhart and Pera [14] studied the effects of mass transfer on a steady free convection flow past a semiinfinite vertical plate by the similarity method and it was assumed that the concentration level of the diffusing species in fluid medium was very low. This assumption enabled them to neglect the diffusion-thermo and thermo-diffusion effects as well as the interfacial velocity at the wall due to species diffusion. Following this assumption, Das et al. [15] investigated the effects of simultaneous heat and mass transfer on free convection flow past an infinite vertical plate under different physical situations.

Recently, the unsteady MHD heat and mass transfer free convection flow of a polar fluid past a vertical moving porous plate in a porous medium with heat generation and thermal diffusion has been studied by Saxena and Dubey [16]. Raveendra Babu et al. [17] studied diffusion-thermo and radiation effects on $\mathrm{MHD}$ free convective heat and mass transfer flow past an infinite vertical plate in the presence of a chemical reaction of first order. Sudhakar et al. [18] discussed chemical reaction effect on an unsteady MHD free convection flow past an infinite vertical accelerated plate with constant heat flux, thermal diffusion, and diffusion thermo.

In the present paper, the flow of an electrically conducting viscous incompressible fluid in a vertical porous channel formed by two vertical parallel porous plates in the presence of a transverse magnetic field is studied. Further, the mass transfer phenomena considered in this problem is associated with chemically reacting species. The objective of the present study is to extend the work of Jha and Ajibade [1] by incorporating the permeability and the magnetic field effect as well as chemical reaction on the flow, heat and mass transfer phenomena.

\section{Mathematical Formulation}

Let us consider the free convective heat and mass transfer MHD flow of a viscous incompressible fluid in a vertical porous channel formed by two infinite vertical parallel porous plates in the presence of chemical reaction. A magnetic field of uniform strength $B_{0}$ is applied transversely to the plate. The induced magnetic field is neglected as the magnetic Reynolds number of the flow is taken to be very small. The convection current is induced due to both the temperature and concentration differences. The flow is assumed to be in the $x^{\prime}$-direction which is taken to be vertically upward along the channel walls and $y^{\prime}$-axis is taken to be normal to the plates that are $h$ distance apart.

Under the usual Boussinesq's approximations, the governing equations for flow are given by

$$
\begin{aligned}
\frac{\partial u^{\prime}}{\partial t^{\prime}}= & \nu \frac{\partial^{2} u^{\prime}}{\partial y^{\prime 2}}+g \beta\left(T^{\prime}-T_{0}\right) \\
& +g \beta^{*}\left(C^{\prime}-C_{0}\right)-\frac{\sigma B_{0}^{2} u^{\prime}}{\rho}-\frac{\nu}{K_{p}^{\prime}} u^{\prime}, \\
\frac{\partial C^{\prime}}{\partial t^{\prime}}= & D \frac{\partial^{2} C^{\prime}}{\partial y^{\prime 2}}-K_{c}^{\prime}\left(C^{\prime}-C_{0}\right), \\
\frac{\partial T^{\prime}}{\partial t^{\prime}}= & \alpha \frac{\partial^{2} T^{\prime}}{\partial y^{\prime 2}}+D_{1} \frac{\partial^{2} C^{\prime}}{\partial y^{\prime 2}},
\end{aligned}
$$

where $\nu, g, \beta, \beta^{*}, \sigma, B_{0}, \rho, D, K_{c}^{\prime}, \alpha, D_{1}$, and $K_{p}^{\prime}$ are kinematic viscosity, acceleration due to gravity, coefficient of thermal expansion, coefficient of mass expansion, electrical conductivity of the fluid, uniform magnetic field, density of the fluid, chemical molecular diffusivity, the dimensional chemical reaction parameter in the diffusion equation, thermal diffusivity, the dimensional coefficient of the diffusion-thermo effect, and the permeability of the medium, respectively.

Initial and boundary conditions of the problem are

$$
\begin{aligned}
& t^{\prime} \leq 0: u^{\prime}\left(y^{\prime}, t^{\prime}\right)=0, \quad T^{\prime}\left(y^{\prime}, t^{\prime}\right)=T_{0}, \quad C^{\prime}\left(y^{\prime}, t^{\prime}\right)=C_{0} \\
& t^{\prime}>0: \begin{array}{lll}
u^{\prime}\left(o, t^{\prime}\right)=0, & T^{\prime}\left(0, t^{\prime}\right)=T_{w}, & C^{\prime}\left(0, t^{\prime}\right)=C_{w} \\
u^{\prime}\left(h, t^{\prime}\right)=0, & T^{\prime}\left(h, t^{\prime}\right)=T_{0}, & C^{\prime}\left(h, t^{\prime}\right)=C_{0} .
\end{array}
\end{aligned}
$$

We now introduce the following dimensionless quantities:

$$
\begin{gathered}
y=\frac{y^{\prime}}{h}, \quad t=\frac{t^{\prime} v}{h^{2}}, \quad u=\frac{u^{\prime} v}{g \beta h^{2}\left(T_{w}-T_{0}\right)}, \\
P_{r}=\frac{\nu}{\alpha}, \quad S_{c}=\frac{\nu}{D}, \\
T=\frac{T^{\prime}-T_{0}}{T_{w}-T_{0}}, \quad C=\frac{C^{\prime}-C_{0}}{C_{w}-C_{0}}, \\
N=\frac{\beta^{*}\left(C_{w}-C_{0}\right)}{\beta\left(T_{w}-T_{0}\right)}, \\
D^{*}=\frac{D_{1}\left(C_{w}-C_{0}\right)}{\alpha\left(T_{w}-T_{0}\right)}, \quad M=\frac{\sigma B_{0}^{2} h^{2}}{\rho \nu}, \\
K_{c}=\frac{K_{c}^{*} h^{2}}{v}, \quad K_{p}=\frac{K_{P}^{\prime}}{h^{2}} .
\end{gathered}
$$


Here $P_{r}, S_{c}, N, D^{*}, M, K_{c}$, and $K_{p}$ are the Prandtl number, the Schmidt number, the Sustentation parameter, the Dufour number, the magnetic field parameter, the chemical reaction parameter, and the Porosity parameter, respectively.

The nondimensional form of (1) is given by

$$
\begin{aligned}
& \frac{\partial u}{\partial t}=\frac{\partial^{2} u}{\partial y^{2}}+N C+T-\left(M+\frac{1}{K_{p}}\right) u, \\
& \frac{\partial C}{\partial t}=\frac{1}{S_{c}} \frac{\partial^{2} C}{\partial y^{2}}-K_{c} C, \\
& \frac{\partial T}{\partial t}=\frac{1}{p_{r}} \frac{\partial^{2} T}{\partial y^{2}}+\frac{D^{*}}{p_{r}} \frac{\partial^{2} C}{\partial y^{2}} .
\end{aligned}
$$

Subject to boundary conditions,

$$
\begin{aligned}
& t \leq 0: u(y, t)=0, \quad T(y, t)=0, \quad C(y, t)=0, \\
& t \leq 0: \begin{array}{lll}
u(0, t)=0, & T(0, t)=1, & C(0, t)=1, \\
u(1, t)=0, & T(1, t)=0, & C(1, t)=0 .
\end{array}
\end{aligned}
$$

\section{Solution of the Problem}

Applying Laplace transform to (5) with boundary condition (6), we get

$$
\begin{gathered}
\frac{d^{2} \bar{C}}{d y^{2}}-S_{c}\left(s+K_{c}\right) \bar{C}=0, \\
\frac{d^{2} \bar{T}}{d y^{2}}-s P_{r} \bar{T}=S_{c} D^{*}\left(s+K_{c}\right) \bar{C}, \\
s \bar{u}=\frac{d^{2} \bar{u}}{d y^{2}}+N \bar{C}+\bar{T}-\left(M+\frac{1}{K_{p}}\right) \bar{u} .
\end{gathered}
$$

The boundary condition (6) becomes

$$
\begin{array}{ll}
\bar{C}(0, s)=\frac{1}{s}, & \bar{C}(1, s)=0, \\
\bar{T}(0, s)=\frac{1}{s}, & \bar{T}(1, s)=0, \\
\bar{u}(0, s)=0, & \bar{u}(1, s)=0 .
\end{array}
$$

Applying inverse Laplace transform to (7) and (8) subject to boundary condition (9), following Debanath and Bhatta [19], and using the formula

$$
\begin{array}{r}
L^{-1}\left(\frac{e^{-b \sqrt{s+a}}}{s}\right)=\frac{1}{2}\left\{e^{-b \sqrt{a}} \operatorname{erfc}\left(\frac{b}{2 \sqrt{t}}-\sqrt{a t}\right)\right. \\
\left.+e^{b \sqrt{a}} \operatorname{erfc}\left(\frac{b}{2 \sqrt{t}}+\sqrt{a t}\right)\right\} .
\end{array}
$$

The solutions of (5) subject to the boundary conditions (6) are obtained by Laplace transform technique.
Case $1\left(P_{r} \neq 1, S_{c} \neq 1\right)$. The solutions of (5) subject to boundary conditions (6) are given by

$$
\begin{gathered}
C=\sum_{n=0}^{\infty}\left[f\left(K_{c}, a_{n} \sqrt{S_{c}}, 1,0, t\right)-f\left(K_{c}, b_{n} \sqrt{S_{c}}, 1,0, t\right)\right] \\
T=A_{1} \sum_{n=0}^{\infty}\left[f\left(0, a_{n} \sqrt{P_{r}}, P_{r}-S_{c}, S_{c} K_{c}, t\right)\right. \\
\left.-f\left(0, b_{n} \sqrt{P_{r}}, P_{r}-S_{c}, S_{c} K_{c}, t\right)\right] \\
-A_{1} \sum_{n=0}^{\infty}\left[f\left(K_{c}, a_{n} \sqrt{S_{c}}, P_{r}-S_{c}, S_{c} K_{c}, t\right)\right. \\
\left.-f\left(K_{c}, b_{n} \sqrt{S_{c}}, P_{r}-S_{c}, S_{c} K_{c}, t\right)\right] \\
+\left(D^{*}+1\right) \sum_{n=0}^{\infty}\left[f\left(0, a_{n} \sqrt{P_{r}}, 1,0, t\right)\right. \\
\left.-f\left(0, b_{n} \sqrt{P_{r}}, 1,0, t\right)\right]-D^{*} C
\end{gathered}
$$

$u=A_{2} \sum_{n=0}^{\infty}\left[f\left(M+\frac{1}{K_{p}}, a_{n}, 1-S_{c}, S_{c} K_{c}-M-\frac{1}{K_{p}}, t\right)\right.$

$$
\begin{array}{r}
\left.-f\left(M+\frac{1}{K_{p}}, b_{n}, 1-S_{c}, S_{c} K_{c}-M-\frac{1}{K_{p}}, t\right)\right] \\
-A_{2} \sum_{n=0}^{\infty}\left[f\left(K_{c}, a_{n} \sqrt{S_{c}}, 1-S_{c}, S_{c} K_{c}-M-\frac{1}{K_{p}}, t\right)\right. \\
\left.-f\left(K_{c}, b_{n} \sqrt{S_{c}}, 1-S_{c}, S_{c} K_{c}-M-\frac{1}{K_{p}}, t\right)\right]
\end{array}
$$$$
+A_{3} \sum_{n=0}^{\infty}\left[f\left(M+\frac{1}{K_{p}}, a_{n}, 1-P_{r},-M-\frac{1}{K_{p}}, t\right)\right.
$$$$
\left.-f\left(M+\frac{1}{K_{p}}, b_{n}, 1-P_{r},-M-\frac{1}{K_{p}}, t\right)\right]
$$$$
-A_{3} \sum_{n=0}^{\infty}\left[f\left(K_{c}, a_{n} \sqrt{P_{r}}, 1-P_{r},-M-\frac{1}{K_{p}}, t\right)\right.
$$$$
\left.-f\left(K_{c}, b_{n} \sqrt{P_{r}}, 1-P_{r},-M-\frac{1}{K_{p}}, t\right)\right]
$$$$
+2 A_{4} \sum_{n=0}^{\infty}\left[f\left(M+\frac{1}{K_{p}}, a_{n}, P_{r}-S_{c}, S_{c} K_{c}, t\right)\right.
$$$$
\left.-f\left(M+\frac{1}{K_{p}}, b_{n}, P_{r}-S_{c}, S_{c} K_{c}, t\right)\right]
$$

$$
-A_{4} \sum_{n=0}^{\infty}\left[f\left(K_{c}, a_{n} \sqrt{S_{c}}, P_{r}-S_{c}, S_{c} K_{c}, t\right)\right.
$$

$$
\left.-f\left(K_{c}, b_{n} \sqrt{S_{c}}, P_{r}-S_{c}, S_{c} K_{c}, t\right)\right]
$$




$$
\begin{aligned}
& -A_{4} \sum_{n=0}^{\infty}\left[f\left(0, a_{n} \sqrt{P_{r}}, P_{r}-S_{c}, S_{c} K_{c}, t\right)\right. \\
& \left.-f\left(0, b_{n} \sqrt{S_{c}}, P_{r}-S_{c}, S_{c} K_{c}, t\right)\right] \\
& +\left(A_{5}-A_{6}\right) \sum_{n=0}^{\infty}\left[f\left(M+\frac{1}{K_{p}}, a_{n}, 1,0, t\right)\right. \\
& \left.-f\left(M+\frac{1}{K_{p}}, b_{n}, 1,0, t\right)\right] \\
& +A_{6} \sum_{n=0}^{\infty}\left[f\left(0, a_{n} \sqrt{P_{r}}, 1,0, t\right)-f\left(0, b_{n} \sqrt{P_{r}}, 1,0, t\right)\right]
\end{aligned}
$$

where $a_{n}=2 n+y$ and $b_{n}=2 n+2-y$.

The functions $f$ and erfc are given in Appendix A. The constants $A_{1}, A_{2}, A_{3}, A_{4}, A_{5}$, and $A_{6}$ are given in Appendix B.

The rate of mass transfer (Sh), the rate of heat transfer $(\mathrm{Nu})$, and the skin function $(\tau)$ at the walls of the channel are obtained as follows:

Sherwood Number (Sh). Consider

$$
\begin{aligned}
& \mathrm{Sh}_{0}=\left.\frac{\partial C}{\partial y}\right|_{y=0} \\
& =-\sqrt{S_{c}} \sum_{n=0}^{\infty}\left[g\left(K_{c}, 2 n \sqrt{S_{c}}, 1,0, t\right)\right. \\
& \left.+g\left(K_{c},(2 n+2) \sqrt{S_{c}}, 1,0, t\right)\right], \\
& \mathrm{Sh}_{1}=\left.\frac{\partial C}{\partial y}\right|_{y=1}=2 \sqrt{S_{c}} \sum_{n=0}^{\infty}\left[g\left(K_{c},(2 n+1) \sqrt{S_{c}}, 1,0, t\right)\right] \text {. }
\end{aligned}
$$

Nusselt Number (Nu). Consider

$$
\begin{aligned}
& \mathrm{Nu}_{0}=-\left.\frac{\partial T}{\partial y}\right|_{y=0} \\
& =A_{1} \sqrt{S_{c}} \sum_{n=0}^{\infty}\left[g\left(K_{c}, 2 n \sqrt{S_{c}}, P_{r}-S_{c}, S_{c} K_{c}, t\right)\right. \\
& \left.+g\left(K_{c},(2 n+2) \sqrt{S_{c}}, P_{r}-S_{c}, S_{c} K_{c}, t\right)\right], \\
& -A_{1} \sqrt{P_{r}} \sum_{n=0}^{\infty}\left[g\left(0,2 n \sqrt{P_{r}}, P_{r}-S_{c}, S_{c} K_{c}, t\right)\right. \\
& \left.+g\left(0,(2 n+2) \sqrt{P_{r}}, P_{r}-S_{c}, S_{c} K_{c}, t\right)\right] \\
& -\left(D^{*}+1\right) \sqrt{P_{r}} \sum_{n=0}^{\infty}\left[g\left(0,2 n \sqrt{P_{r}}, 1,0, t\right)\right. \\
& \left.+g\left(0,(2 n+2) \sqrt{P_{r}}, 1,0, t\right)\right]
\end{aligned}
$$

$-D^{*} \mathrm{Sh}_{0}$

$$
\begin{aligned}
& \mathrm{Nu}_{1}=\left.\frac{\partial T}{\partial y}\right|_{y=1} \\
& =2 A_{1} \sqrt{P_{r}} \sum_{n=0}^{\infty}\left[g\left(0,(2 n+1) \sqrt{P_{r}}, P_{r}-S_{c}, S_{c} K_{c}, t\right)\right] \\
& -2 A_{1} \sqrt{S_{c}} \sum_{n=0}^{\infty}\left[g\left(K_{c},(2 n+1) \sqrt{S_{c}}, P_{r}-S_{c}, S_{c} K_{c}, t\right)\right] \\
& +2\left(D^{*}+1\right) \sqrt{P_{r}} \sum_{n=0}^{\infty}\left[g\left(0,(2 n+1) \sqrt{P_{r}}, 1,0, t\right)\right] \\
& -D^{*} \mathrm{Sh}_{1} \text {. }
\end{aligned}
$$

Skin Friction $(\tau)$. Consider

$$
\begin{aligned}
& \tau_{0}=-\left.\frac{\partial u}{\partial y}\right|_{y=0} \\
& =A_{2} \sum_{n=0}^{\infty}\left[g\left(M+\frac{1}{K_{p}}, 2 n, 1-S_{c}, S_{c} K_{c}-M-\frac{1}{K_{p}}, t\right)\right. \\
& +g\left(M+\frac{1}{K_{p}}, 2 n+2,1-S_{c}, S_{c} K_{c}\right. \\
& \left.\left.-M-\frac{1}{K_{p}}, t\right)\right] \\
& -A_{2} \sqrt{S_{c}} \sum_{n=0}^{\infty}\left[g\left(K_{c}, 2 n \sqrt{S_{c}}, 1-S_{c}, S_{c} K_{c}-M-\frac{1}{K_{p}}, t\right)\right. \\
& +g\left(K_{c},(2 n+2) \sqrt{S_{c}}, 1-S_{c}, S_{c} K_{c}\right. \\
& \left.\left.-M-\frac{1}{K_{p}}, t\right)\right] \\
& +A_{3} \sum_{n=0}^{\infty}\left[g\left(M+\frac{1}{K_{p}}, 2 n, 1-P_{r},-M-\frac{1}{K_{p}}, t\right)\right. \\
& +g\left(M+\frac{1}{K_{p}}, 2 n+2,1-P_{r}\right. \\
& \left.\left.-M-\frac{1}{K_{p}}, t\right)\right] \\
& -A_{3} \sqrt{P_{r}} \sum_{n=0}^{\infty}\left[g\left(K_{c}, 2 n \sqrt{P_{r}}, 1-P_{r}-M-\frac{1}{K_{p}}, t\right)\right. \\
& +g\left(K_{c},(2 n+2) \sqrt{P_{r}}, 1-P_{r}\right. \\
& \left.\left.-M-\frac{1}{K_{p}}, t\right)\right]
\end{aligned}
$$




$$
\begin{aligned}
& +2 A_{4} \sum_{n=0}^{\infty}\left[g\left(M+\frac{1}{K_{p}}, 2 n, P_{r}-S_{c}, S_{c} K_{c}, t\right)\right. \\
& \left.+g\left(M+\frac{1}{K_{p}}, 2 n+2, P_{r}-S_{c}, S_{c} K_{c}, t\right)\right] \\
& -A_{4} \sqrt{S_{C}} \sum_{n=0}^{\infty}\left[g\left(K_{c}, 2 n \sqrt{S_{c}}, P_{r}-S_{c}, S_{c} K_{c}, t\right)\right. \\
& \left.+g\left(K_{c},(2 n+2) \sqrt{S_{c}}, P_{r}-S_{c}, S_{c} K_{c}, t\right)\right] \\
& -A_{4} \sqrt{P_{r}} \sum_{n=0}^{\infty}\left[g\left(0,2 n \sqrt{P_{r}}, P_{r}-S_{c}, S_{c} K_{c}, t\right)\right. \\
& \left.+g\left(0,(2 n+2) \sqrt{P_{r}}, P_{r}-S_{c}, S_{c} K_{c}, t\right)\right] \\
& +\left(A_{5}-A_{6}\right) \sum_{n=0}^{\infty}\left[g\left(M+\frac{1}{K_{p}}, 2 n, 1,0, t\right)\right. \\
& \left.+g\left(M+\frac{1}{K_{p}}, 2 n+2,1,0, t\right)\right] \\
& +A_{5} \mathrm{Sh}_{0}+A_{6} \sqrt{P_{r}} \sum_{n=0}^{\infty}\left[g\left(0,2 n \sqrt{P_{r}}, 1,0, t\right)\right. \\
& \left.+g\left(0,(2 n+2) \sqrt{P_{r}}, 1,0, t\right)\right] \\
& \tau_{1}=-\left.\frac{\partial u}{\partial y}\right|_{y=1} \\
& =-2 A_{2} \sum_{n=0}^{\infty}\left[g \left(M+\frac{1}{K_{p}}, 2 n+1,1-S_{c}, S_{c} K_{c}\right.\right. \\
& \left.\left.-M-\frac{1}{K_{p}}, t\right)\right] \\
& +2 A_{2} \sqrt{S_{c}} \sum_{n=0}^{\infty}\left[g \left(K_{c},(2 n+1) \sqrt{S_{c}}, 1-S_{c}, S_{c} K_{c}\right.\right. \\
& -M, t)] \\
& -2 A_{3} \sum_{n=0}^{\infty}\left[g\left(M+\frac{1}{K_{p}}, 2 n+1,1-P_{r},-M-\frac{1}{K_{p}}, t\right)\right] \\
& +2 A_{3} \sqrt{P_{r}} \sum_{n=0}^{\infty}\left[g \left(K_{c},(2 n+1) \sqrt{P_{r}}, 1-P_{r},-M\right.\right. \\
& \left.\left.-\frac{1}{K_{p}}, t\right)\right] \\
& -4 A_{4} \sum_{n=0}^{\infty}\left[g\left(M+\frac{1}{K_{p}}, 2 n+1, P_{r}-S_{c}, S_{c} K_{c}, t\right)\right] \\
& +2 A_{4} \sqrt{S_{c}} \sum_{n=0}^{\infty}\left[g\left(K_{c},(2 n+1) \sqrt{S_{c}}, P_{r}-S_{c}, S_{c} K_{c}, t\right)\right] \\
& +2 A_{4} \sqrt{P_{r}} \sum_{n=0}^{\infty}\left[g\left(0,(2 n+1) \sqrt{P_{r}}, P_{r}-S_{c}, S_{c} K_{c}, t\right)\right]
\end{aligned}
$$

$$
\begin{aligned}
& -2\left(A_{5}-A_{6}\right) \sum_{n=0}^{\infty}\left[g\left(M+\frac{1}{K_{p}}, 2 n+1,1,0, t\right)\right] \\
& -2 A_{6} \sqrt{P_{r}} \sum_{n=0}^{\infty}\left[g\left(0,(2 n+1) \sqrt{P_{r}}, 1,0, t\right)\right]+A_{5} \mathrm{Sh}_{1},
\end{aligned}
$$

where the function $g$ is given in Appendix A.

The mass flux (volumetric flow rate) for the problem is given by

$$
\mathrm{Q}=\int_{0}^{1} u d y .
$$

This is computed by using the trapezoidal rule for numerical integration.

Case $2\left(P_{r}=S_{c}=1\right)$. The solutions of (5) subject to boundary conditions (6) are given by

$$
\begin{gathered}
C=\sum_{n=0}^{\infty}\left[f\left(0, a_{n}, 1,0, t\right)-f\left(0, b_{n}, 1,0, t\right)\right] \\
T=\frac{D^{*}\left(1-e^{-K_{c} t}\right)}{2 K_{c} t \sqrt{\pi t}} \sum_{n=0}^{\infty}\left[a_{n} \exp \left(-\frac{a_{n}^{2}}{4 t}\right)-b_{n} \exp \left(-\frac{b_{n}^{2}}{4 t}\right)\right] \\
+\left(D^{*}+1\right) \sum_{n=0}^{\infty}\left[f\left(0, a_{n}, 1,0, t\right)-f\left(0, b_{n}, 1,0, t\right)\right]-D^{*} C \\
u=A_{8} C+\frac{D^{*}}{2 t \sqrt{\pi t}}\left[\frac{\exp \left(-K_{c} t\right)}{K_{c}\left(K_{c}-M-\left(1 / K_{p}\right)\right)}\right. \\
+\frac{1}{\left(M+\left(1 / K_{p}\right)\right) K_{c}} \\
+\sum_{n=0}^{\infty}\left[a_{n} \exp \left(-\frac{a_{n}^{2}}{4 t}\right)-b_{n} \exp \left(-\frac{b_{n}^{2}}{4 t}\right)\right] \\
+A_{6} \sum_{n=0}^{\infty}\left[f\left(0, a_{n}, 1,0, t\right)-f\left(0, b_{n}, 1,0, t\right)\right],
\end{gathered}
$$

where $A_{7}$ and $A_{8}$ are given in Appendix B.

In this case, the rate of mass transfer (Sh), the rate of heat transfer $(\mathrm{Nu})$, and the skin frictions $(\tau)$ on the walls of the channel are obtained as follows:

Sherwood Number. Consider

$$
\begin{aligned}
\mathrm{Sh}_{0} & =-\left.\frac{\partial C}{\partial y}\right|_{y=0} \\
& =-\sum_{n=0}^{\infty}\left[g\left(K_{c}, 2 n, 1,0, t\right)-g\left(K_{c}, 2 n+2,1,0, t\right)\right], \\
\mathrm{Sh}_{1} & =-\left.\frac{\partial C}{\partial y}\right|_{y=1}=2 \sum_{n=0}^{\infty}\left[g\left(K_{c}, 2 n+1,1,0, t\right)\right] .
\end{aligned}
$$


Nusselt Number. Consider

$$
\begin{aligned}
& \mathrm{Nu}_{0}=-\left.\frac{\partial T}{\partial y}\right|_{y=0} \\
& =-\frac{D^{*}\left[1-\exp \left(-K_{c} t\right)\right]}{2 K_{c} t \sqrt{\pi t}} \\
& \times\left[\sum_{n=0}^{\infty}\left\{\exp \left(-\frac{n^{2}}{t}\right)+\exp \left(-\frac{(n+1)^{2}}{t}\right)\right\}\right. \\
& -\frac{2}{t} \sum_{n=0}^{\infty}\left\{n^{2} \exp \left(-\frac{n^{2}}{t}\right)\right. \\
& \left.\left.+(n+1)^{2} \exp \left(-\frac{(n+1)^{2}}{t}\right)\right\}\right] \\
& -\left(D^{*}+1\right) \sum_{n=0}^{\infty}[g(0,2 n, 1,0, t)+g(0,2 n+2,1,0, t)] \\
& +D^{*} \mathrm{Sh}_{0} \text {, } \\
& \mathrm{Nu}_{1}=-\left.\frac{\partial T}{\partial y}\right|_{y=0} \\
& =\frac{D^{*}\left[1-\exp \left(-K_{c} t\right)\right]}{2 K_{c} t \sqrt{\pi t}} \\
& \times\left[2 \sum_{n=0}^{\infty} \exp \left(-\frac{(2 n+1)^{2}}{4 t}\right)\right. \\
& \left.-\frac{1}{t} \sum_{n=0}^{\infty}(2 n+1)^{2} \exp \left(-\frac{(2 n+1)^{2}}{4 t}\right)\right] \\
& +2\left(D^{*}+1\right) \sum_{n=0}^{\infty}[g(0,2 n+1,1,0, t)]-D^{*} \mathrm{Sh}_{1} \text {. }
\end{aligned}
$$

Skin Friction. Consider

$$
\begin{aligned}
\tau_{0}= & -\left.\frac{\partial u}{\partial y}\right|_{y=0} \\
= & A_{8} S_{0}+\frac{D^{*}}{2 t \sqrt{\pi t}} \\
\times & {\left[\frac{\exp \left(-K_{c} t\right)}{K_{c}\left(K_{c}-M-\left(1 / K_{p}\right)\right)}+\frac{1}{K_{c}\left(M+\left(1 / K_{p}\right)\right)}\right.} \\
& \left.-\frac{\exp (-M t)}{\left(M+\left(1 / K_{p}\right)\right)\left(K_{c}-M-\left(1 / K_{p}\right)\right)}\right] \\
\times & {\left[\sum_{n=0}^{\infty}\left\{\exp \left(-\frac{n^{2}}{t}\right)+\exp \left(-\frac{(n+1)^{2}}{t}\right)\right\}\right.} \\
& -\frac{2}{t} \sum_{n=0}^{\infty}\left\{n^{2} \exp \left(-\frac{n^{2}}{t}\right)\right.
\end{aligned}
$$

$$
\begin{aligned}
& \left.\left.+(n+1)^{2} \exp \left(-\frac{(n+1)^{2}}{t}\right)\right\}\right] \\
& +A_{6} \sum_{n=0}^{\infty}[g(0,2 n, 1,0, t)+g(0,2 n+2,1,0, t)] \\
& -A_{7} \sum_{n=0}^{\infty}\left[g\left(M+\frac{1}{K_{p}}, 2 n, 1,0, t\right)\right. \\
& \left.+g\left(M+\frac{1}{K_{p}}, 2 n+2,1,0, t\right)\right] \\
& \tau_{1}=-\left.\frac{\partial u}{\partial y}\right|_{y=1} \\
& =A_{8} \mathrm{Sh}_{1}+\frac{D^{*}}{t \sqrt{\pi t}} \\
& \times\left[\frac{\exp \left(-M-\left(1 / K_{p}\right)\right) t}{\left(M+\left(1 / K_{p}\right)\right)\left(K_{c}-M-\left(1 / K_{p}\right)\right)}\right. \\
& \left.-\frac{1}{K_{c}\left(M+\left(1 / K_{p}\right)\right)}-\frac{\exp \left(-K_{c} t\right)}{K_{c}\left(K_{c}-M-\left(1 / K_{p}\right)\right)}\right] \\
& \times \sum_{n=0}^{\infty}\left[\exp \left(-\frac{(2 n+1)^{2}}{t}\right)\right. \\
& \left.-2 \frac{(2 n+1)^{2}}{t} \exp \left(-\frac{(2 n+1)^{2}}{t}\right)\right] \\
& -2 A_{6} \sum_{n=0}^{\infty}[g(0,2 n+1,1,0, t)] \\
& \times 2 A_{7} \sum_{n=0}^{\infty}\left[g\left(M+\frac{1}{K_{p}}, 2 n+1,1,0, t\right)\right] \text {. }
\end{aligned}
$$

Steady State. Setting $\partial u / \partial \mathrm{t}=0, \partial T / \partial \mathrm{t}=0$ and $\partial C / \partial \mathrm{t}=0$ in (5), the steady state of the problem is obtained as

$$
\begin{aligned}
& \frac{d^{2} u}{d y^{2}}+N C+T-\left(M+\frac{1}{K_{p}}\right) u=0, \frac{d^{2} C}{d y^{2}}-S_{c} K_{c} C=0, \\
& \frac{d^{2} T}{d y^{2}}+D^{*} \frac{d^{2} C}{d y^{2}}=0 .
\end{aligned}
$$

The solutions of (21) subject to boundary conditions (6) are given by

$$
\begin{gathered}
C=\frac{\sinh \left(\sqrt{S_{c} K_{c}}(1-y)\right)}{\sinh \left(\sqrt{S_{c} K_{c}}\right)}, \\
T=\left(D^{*}+1\right)(1-y)-D^{*} C,
\end{gathered}
$$




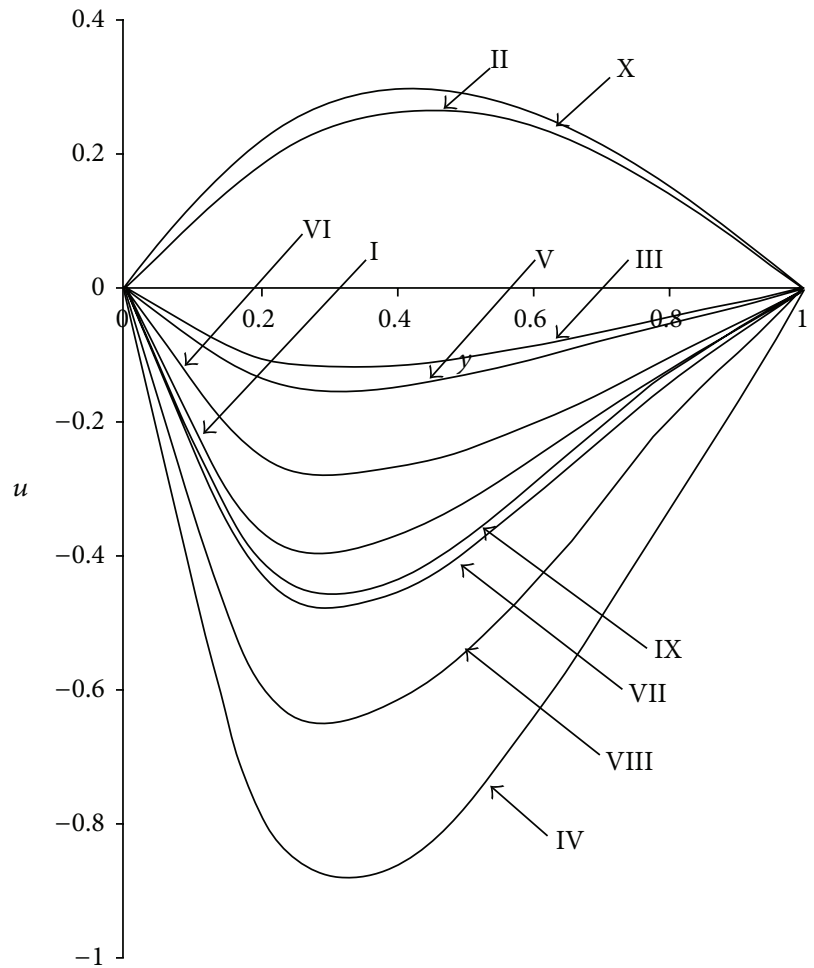

\begin{tabular}{|c|c|c|c|c|c|c|c|c|}
\hline Curve & $t$ & $M$ & $S_{c}$ & $K_{c}$ & $N$ & $P_{r}$ & $D^{*}$ & $K_{p}$ \\
\hline I & 0.4 & 0.5 & 0.22 & 2 & 2 & 1.8 & 2 & 100 \\
\hline II & 0.2 & 0.5 & 0.22 & 2 & 2 & 1.8 & 2 & 100 \\
\hline III & 0.4 & 2 & 0.22 & 2 & 2 & 1.8 & 2 & 100 \\
\hline IV & 0.4 & 0.5 & 0.3 & 2 & 2 & 1.8 & 2 & 100 \\
\hline V & 0.4 & 0.5 & 0.22 & 1 & 2 & 1.8 & 2 & 100 \\
\hline VI & 0.4 & 0.5 & 0.22 & 2 & 4 & 1.8 & 2 & 100 \\
\hline VII & 0.4 & 0.5 & 0.22 & 2 & 2 & 2 & 2 & 100 \\
\hline VIII & 0.4 & 0.5 & 0.22 & 2 & 2 & 1.8 & 4 & 100 \\
\hline IX & 0.4 & 0.5 & 0.22 & 2 & 2 & 1.8 & 2 & 0.5 \\
\hline X & $\begin{array}{c}\text { Steady } \\
\text { state }\end{array}$ & 0.5 & 0.22 & 2 & 4 & - & 2 & 100 \\
\hline
\end{tabular}

FIGURE 1: Velocity profile.

$$
u=A_{9} \frac{\sinh \sqrt{\left(M+\left(1 / K_{p}\right)\right)}(1-y)}{\sinh \sqrt{M+\left(1 / K_{p}\right)}}+A_{5} C+A_{6}(1-y),
$$

where $A_{9}$ is given in Appendix B.

In this case, the Sherwood number (Sh), the Nusselt number $(\mathrm{Nu})$, and the skin friction $(\tau)$ are given by the following:

Sherwood Number (Sh). Consider

$$
\begin{aligned}
\mathrm{Sh}_{0} & =\sqrt{S_{c} K_{c}} \operatorname{coth} \sqrt{S_{c} K_{c}}, \\
S_{1} & =\sqrt{S_{c} K_{c}} \operatorname{cosech} \sqrt{S_{c} K_{c}} .
\end{aligned}
$$

Nusselt Number (Nu). Consider

$$
\begin{gathered}
\mathrm{Nu}_{0}=D^{*}+1+D^{*} \mathrm{Sh}_{0}, \\
\mathrm{Nu}_{1}=-\left(D^{*}+1\right)-D^{*} \mathrm{Sh}_{1} .
\end{gathered}
$$

Skin Friction $(\tau)$. Consider

$$
\begin{aligned}
& \tau_{0}=-A_{9} \sqrt{M+\frac{1}{K_{p}}} \operatorname{coth} \sqrt{M+\frac{1}{K_{p}}}+A_{5} \mathrm{Sh}_{0}-A_{6}, \\
& \tau_{1}=\frac{A_{9} \sqrt{M+\left(1 / K_{p}\right)}}{\sinh \sqrt{M+\left(1 / K_{p}\right)}}-A_{5} \mathrm{Sh}_{1}+A_{6} .
\end{aligned}
$$

Mass Flux. Consider

$$
\begin{aligned}
Q= & A_{9} \sqrt{M+\frac{1}{K_{p}}} \frac{\cosh \sqrt{M+\left(1 / K_{p}\right)}-1}{\sinh \sqrt{M+\left(1 / K_{p}\right)}} \\
& +\frac{A_{5}}{\sqrt{S_{c} K_{c}}} \frac{\cosh \sqrt{S_{c} K_{c}}-1}{\sinh \sqrt{S_{c} K_{c}}}+\frac{A_{6}}{2} .
\end{aligned}
$$

\section{Results and Discussion}

Computations have been carried out by assigning the values to the pertinent parameters characterising the fluids of practical interest. The flow phenomenon is characterized by magnetic parameter $(M)$, chemical reaction parameter $\left(K_{c}\right)$, sustentation parameter $(N)$, Dufour number $\left(D^{*}\right)$, Schmidt number $\left(S_{c}\right)$, Prandtl number $\left(P_{r}\right)$, and porosity parameter $\left(K_{p}\right)$. The effects of various parameters on velocity, temperature, and concentration profiles are shown graphically and in tabulated form for Sherwood number (Sh), Nusselt number $(\mathrm{Nu})$, and skin friction $(\tau)$.

The particular case without magnetic field and porosity $\left(M=0, K_{p} \rightarrow \infty\right)$ can be obtained from (8) which is in good agreement with the work reported earlier [1].

Another interesting point to note is that diffusion in aqueous solution, that is, for higher values of $S_{c}$ gives rise to oscillations in the velocity as well as temperature field in the presence of chemical reaction parameter $\left(K_{c}\right)$.

Figure 1 depicts the velocity profile for various values of pertinent parameters characterising flow fields. The common 


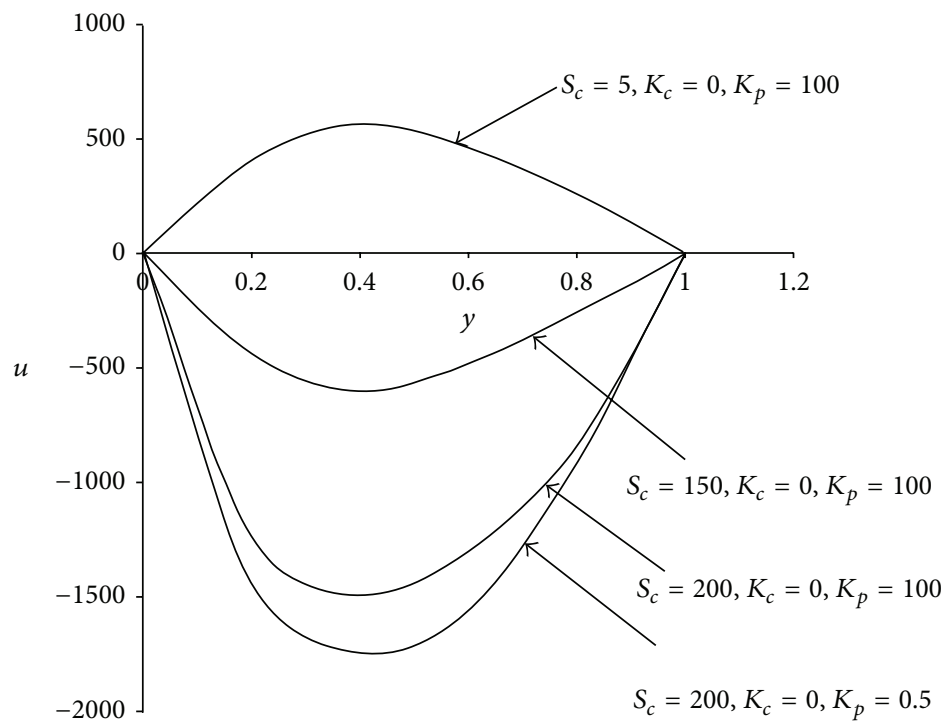

FIGURE 2: Velocity profile for $t=0.4, M=0.5, N=2, P_{r}=7$, and $D^{*}=2$.

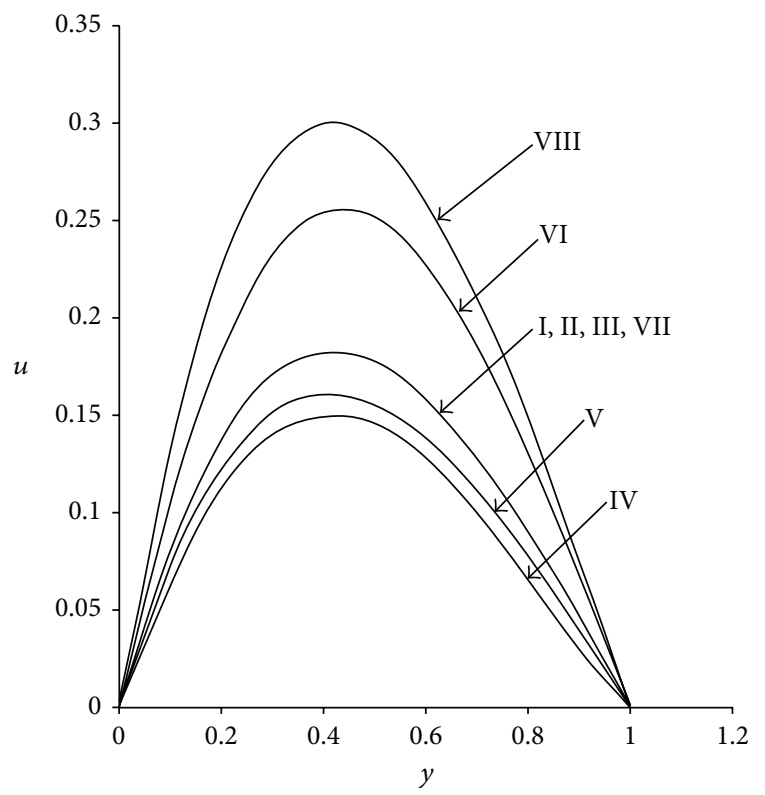

\begin{tabular}{|c|c|c|c|c|c|c|}
\hline Curve & $M$ & $S_{c}$ & $K_{c}$ & $N$ & $D^{*}$ & $K_{p}$ \\
\hline I & 0.5 & 100 & 1 & 2 & 2 & 100 \\
\hline II & 0.5 & 0.22 & 2 & 2 & 2 & 100 \\
\hline III & 0.5 & 152 & 1 & 2 & 2 & 100 \\
\hline IV & 0.5 & 0.22 & 2 & 2 & 2 & 0.5 \\
\hline V & 2 & 0.22 & 2 & 2 & 2 & 100 \\
\hline VI & 0.5 & 0.22 & 2 & 2 & 30 & 100 \\
\hline VII & 0.5 & 0.3 & 1 & 2 & 2 & 100 \\
\hline VIII & 0.5 & 0.22 & 2 & 4 & 2 & 100 \\
\hline
\end{tabular}

Figure 3: Velocity profile (steady state).

feature of the profiles is parabolic. This is evident from curves II and $\mathrm{X}(t=0.2$, Curve II; the steady state curve $\mathrm{X})$ in case of steady state as well as $t \leq 0.3$; the velocity profiles remain positive throughout the flow field. This is evident from curves II and X ( $t=0.2$, Curve II; the steady state curve X). Thus it is concluded that the time span plays a vital role for engendering back flow (Curve I and Curve II).

Further, magnetic parameter $(M)$, chemical reaction parameter $\left(K_{c}\right)$, sustentation parameter $(N)$, and porosity parameter $\left(K_{p}\right)$ decrease the velocity $|u|$ at all points. In all other cases such as $S_{c}, P_{r}$, and $D^{*}$, the reverse effect is observed. If the mass diffusivity becomes greater than the thermal diffusivity (i.e., $N$, the sustentation parameter $>1.0$ ) then the velocity decreases. Moreover, with the stronger magnetic field Lorentz force also reduces the velocity field which is in conformity with the result of Cramer and Pai [20].

An increase in $P_{r}$ leads to decrease in the velocity which suggests that low rate of thermal diffusion leads to increase in the velocity boundary layer thickness.

Figure 2 depicts the velocity distribution in the absence of chemical reaction. It is noteworthy to observe that the back flow occurs for higher values of $S_{c}$; that is, $S_{c}=150$ and $S_{c}=200$ and the permeability of the medium representing the diffusion in aqueous solution in the presence of constant magnetic field and Dufour effect. Further, it is to mention that for $S_{c}<1.0$ and $K_{c}=0.0$, no velocity profile could be graphed/presented. Further, it is to note that heavier species that is, with increasing $S_{c}$ lead to flow reversal. It is most 


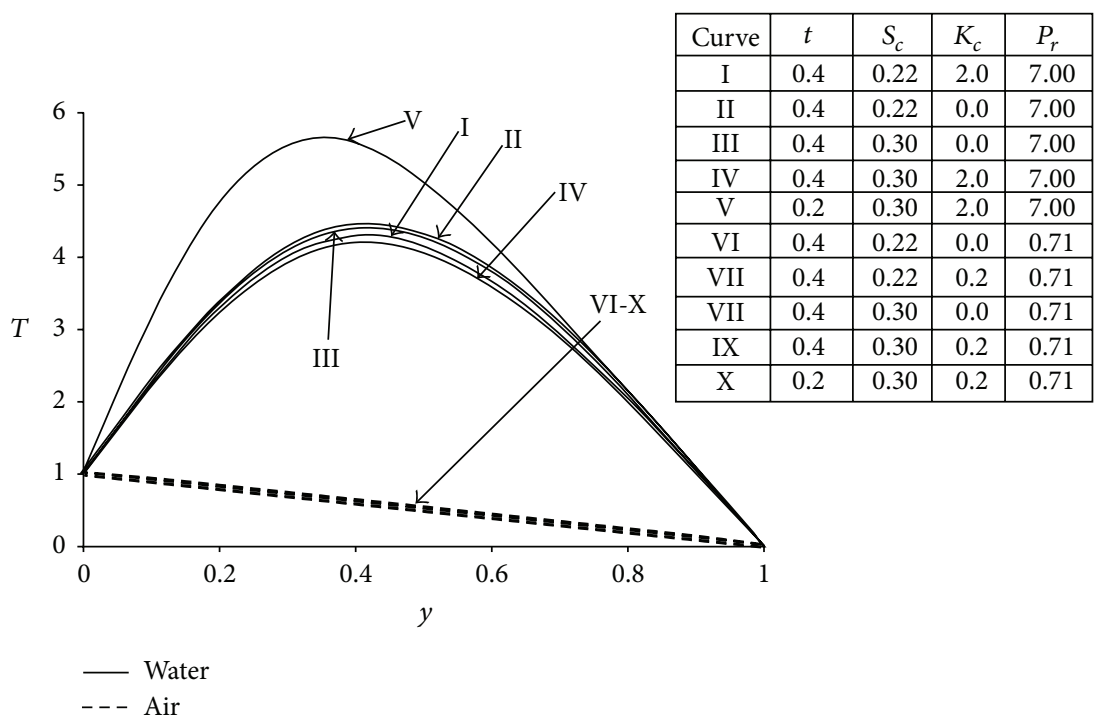

FIgURE 4: Temperature profile for $D^{*}=2.0, P_{r}=7.0$ (water), and $P_{r}=0.71$ (air).

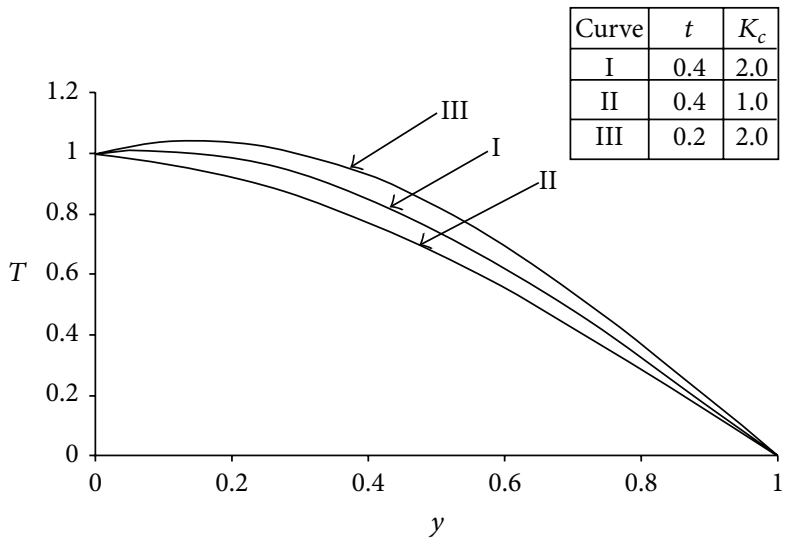

Figure 5: Temperature profile $\left(P_{r}=1, S_{c}=1\right)$.

interesting to note that in the presence of destructive reaction that is, $K_{c}>0$, higher value of $S_{c}$ leads to oscillatory flow and, in case of diffusion in the aqueous solution, it leads to back flow. Thus, this suggests that the back flow is prevented by diffusing lighter species which is in conformity with the results reported earlier by Pop [21], Hossain and Mohammad [22], and Rath et al. [23].

It is also remarked that when $S_{c}$ varies from 0 to 5 the back flow is prevented in the absence of chemical reaction. But in the presence of heavier species the sharp fall of velocity is well marked.

Figure 3 exhibits the steady state velocity profiles indicating no back flow irrespective of higher or lower value of mass transfer coefficient and Dufour effect.

On careful observation, it is revealed that for higher values of Dufour number $D^{*}$ (curve VI), sustentation parameter $N$ (Curve VIII), magnetic parameter $M$ (Curve V), and porosity parameter $K_{p}$ (Curve IV) steady state velocity is effected significantly whereas variation in reaction parameter and Schmidt number produces no change. Thus, it is important to note that mass transfer phenomena with chemical reaction does not affect the steady flow significantly but increase in $M, N, D^{*}$, and $K_{p}$, decrease the velocity, increase the velocity, increase the velocity, and decrease the velocity, respectively. It may be inferred that magnetic parameter, porosity parameter, and Dufour effect produce the same effect irrespective of steady or unsteady state except for sustentation parameter.

Figures 4, 5, and 6 present the temperature distribution. It is seen that for an increase in destructive reaction parameter $\left(K_{c}>0\right)$ temperature increases whereas increase in $P_{r}, t$, and $S_{c}$ reduces the temperature at all points for $P_{r}=7.0$ (water) when $y<0.7$. It is noted that, the slow rate of diffusion leaves the heat energy spread in the fluid mass and it is enhanced for heavier species.

It is observed in case of $P_{r}=0.71$ (air) that the temperature falls sharply at all points with almost linear distribution regardless of other parameters. Thus, it may be pointed out 


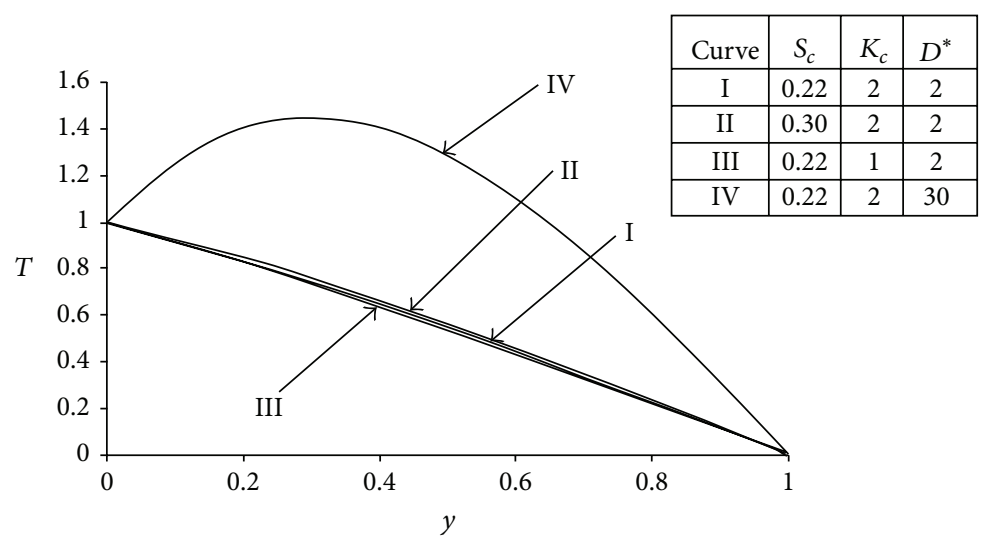

FIGURE 6: Temperature profile (steady state).

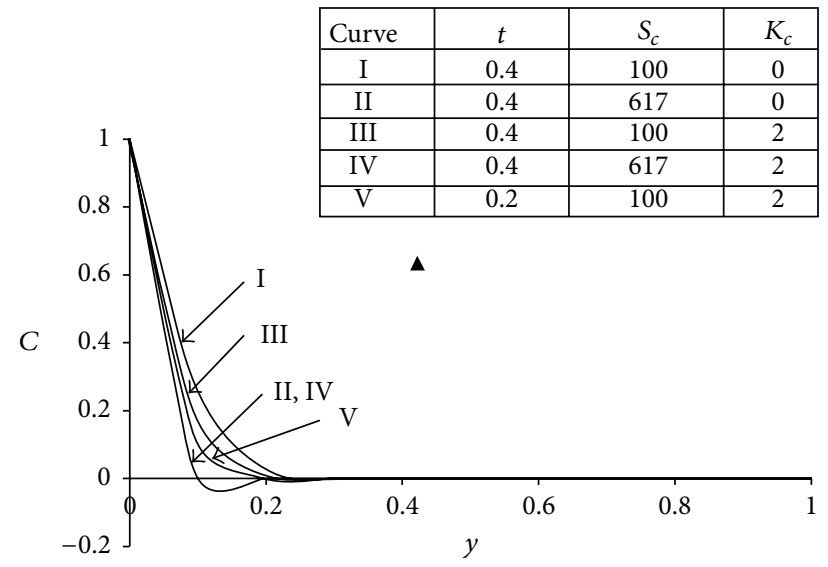

FIGURE 7: Concentration profile.

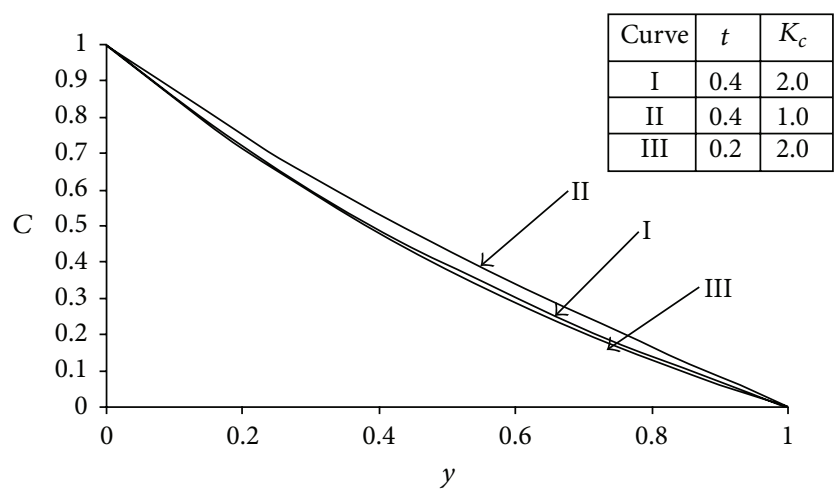

Figure 8: Concentration profile $\left(S_{c}=1\right)$.

that thermal diffusivity property of the fluid plays a vital role in controlling the temperature distribution and hence contributing to thermal boundary layer thickness.

Figure 5 also exhibits the temperature distribution when $P_{r}=1$ and $S_{c}=1$ which means that thermal diffusivity and mass diffusivity are at par. This contributes to the uniform variation/decrease in temperature. Increase in reaction parameter gives rise to increase in temperature whereas allowing for a large span of time reduces it.

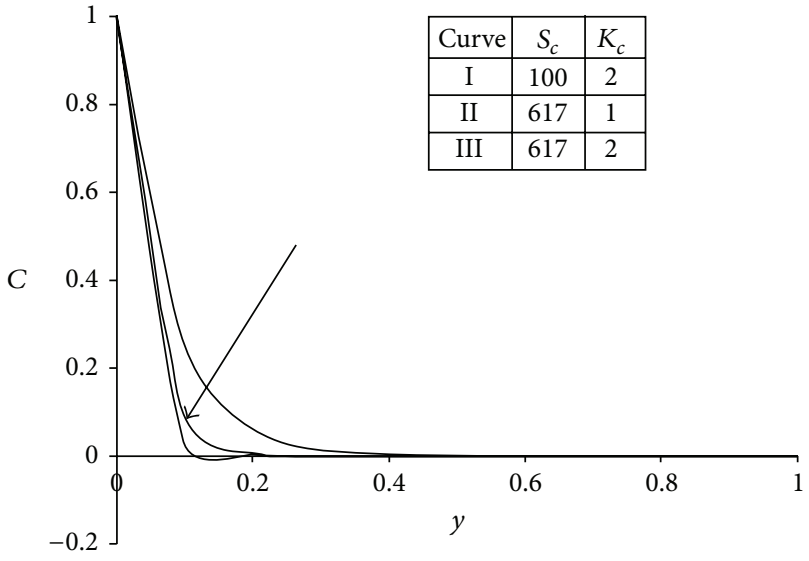

Figure 9: Concentration profile (steady state).

Figure 6 presents the nonlinearity distribution of temperature due to high value of $D^{*}$, that is, for large Dufour effect. Thus, sharp rise and fall of temperature is due to low diffusivity and high Dufour effect.

Figures 7, 8 and 9 exhibit the concentration variation in the flow domain. Sharp fall of concentration is indicated in Figures 7 and 9 along with mass absorption near the plate for very high value of $S_{c}$ (i.e., $S_{c}=617$ ). This means that heavier species give rise to sharp fall of concentration accelerating the process of mass diffusion whereas in case of lighter species the variation is smooth.

Skin friction plays an important role in flow characteristics. It measures the frictional forces encountered at the solid surfaces due to the motion of the fluid. One striking feature of the entries of the skin friction in Table 1, in case of unsteady motion, is that the skin frictions bear same sign in case of lower and upper plate. Further, on careful observation it is revealed that at the lower plate all are negative for $t=0.4$ except for the case when $t=0.2$. Thus, it may be concluded that time span plays an important role to modify the frictional drag due to shear stress at the plates.

Moreover, from (18)-(19) it is clear that the Prandtl number $\left(P_{r}\right)$ has no role to play to affect the velocity, temperature, and concentration fields in the steady flow and hence the skin 
TABLE 1: Skin friction (unsteady case).

\begin{tabular}{lcccccccccc}
\hline Sl. no. & $t$ & $M$ & $S_{c}$ & $K_{c}$ & $N$ & $P_{r}$ & $D^{*}$ & $K_{p}$ & $\tau_{0}$ \\
\hline 1 & 0.4 & 0.5 & 0.22 & 2 & 2 & 1.8 & 2 & 100 & -1.37638 \\
2 & 0.2 & 0.5 & 0.22 & 2 & 2 & 1.8 & 2 & 100 & 0.649408 & 0.466308 \\
3 & 0.4 & 2 & 0.22 & 2 & 2 & 1.8 & 2 & 100 & -0.40403 & -0.12279 \\
4 & 0.4 & 0.5 & 0.3 & 2 & 2 & 1.8 & 2 & 100 & -2.90421 & -0.9827 \\
5 & 0.4 & 0.5 & 0.22 & 1 & 2 & 1.8 & 2 & 100 & -0.46631 & -0.15838 \\
6 & 0.4 & 0.5 & 0.22 & 2 & 4 & 1.8 & 2 & 100 & -0.91633 & -0.28675 \\
7 & 0.4 & 0.5 & 0.22 & 2 & 2 & 2 & 2 & 100 & -1.66428 & -0.3346 \\
8 & 0.4 & 0.5 & 0.22 & 2 & 2 & 1.8 & 4 & 100 & -2.24604 & -0.34433 \\
9 & 0.4 & 0.5 & 0.22 & 2 & 2 & 1.8 & 2 & 0.5 & -1.25513 & -0.2425 \\
\hline
\end{tabular}

TABLE 2: Skin friction (steady case).

\begin{tabular}{lcccccccc}
\hline Sl. no. & $M$ & $S_{c}$ & $K_{c}$ & $N$ & $D^{*}$ & $K_{p}$ & $\tau_{0}$ \\
\hline 1 & 0.5 & 0.22 & 2 & 2 & 2 & 100 & 1.732053 & 0.932515 \\
2 & 2 & 0.22 & 2 & 2 & 2 & 100 & 1.66425 & 1.732052 \\
3 & 0.5 & 0.3 & 2 & 2 & 2 & 100 & 1.73205 & 0.795436 \\
4 & 0.5 & 0.22 & 1 & 2 & 2 & 100 & 0.932515 \\
5 & 0.5 & 0.22 & 2 & 4 & 2 & 100 & 1.664279 \\
6 & 0.5 & 0.22 & 2 & 2 & 4 & 100 & 2.144507 & 0.869287 \\
7 & 0.5 & 0.22 & 2 & 2 & 2 & 0.5 & 1.42312 & 0.524363 \\
\hline
\end{tabular}

TABLE 3: Nusselt number.

\begin{tabular}{lccccccc}
\hline Sl. no. & $t$ & $S_{c}$ & $K_{c}$ & $P_{r}$ & $D^{*}$ & $\mathrm{Nu}_{0}$ & $\mathrm{Nu}_{1}$ \\
\hline 1 & 0.4 & 0.22 & 2 & 7.00 & 2 & -1.37638192 & -1.150368407 \\
2 & 0.2 & 0.22 & 2 & 7.00 & 2 & -2.674621494 & -1.213097004 \\
3 & 0.4 & 0.30 & 2 & 7.00 & 2 & -1.327044822 & -1.110612515 \\
4 & 0.4 & 0.22 & 0 & 7.00 & 2 & -1.455009029 & -1.191753593 \\
5 & 0.4 & 0.22 & 2 & 0.71 & 2 & 0.113935608 & -0.113935608 \\
6 & 0.4 & 0.22 & 2 & 7.00 & 4 & -1.569685558 & -1.279941632 \\
\hline
\end{tabular}

friction. In case of unsteady flow, as $P_{r}$ increases, the shearing stress increases at both the plates.

Considering Tables 1 and 2 for both steady and unsteady cases, it is concluded that an increase in magnetic parameter, porosity parameter, and sustentation parameter leads to reduce the magnitude of frictional drag at both plates. Moreover, an increase in $S_{c}, K_{c}$, and $D^{*}$ leads to enhance the frictional drag at the plates for steady and unsteady flow.

Physically, we may interpret the above results as follows.

Lorentz force and sustentation parameter, that is, ratios of buoyancy effects due to temperature and concentration differences reduce the frictional drag which agrees well with the established result reported earlier. Moreover, presence of heavier species and exothermic reaction and Dufour effect enhance the magnitude of the shearing stress at both plates.

From Table 3 it is seen that an increase in $t, S_{c}$, and $K_{c}$ leads to decrease the Nusselt number at both plates. One interesting point is that rate of heat transfer remains negative for all the parameters at both the plates for aqueous solution $P_{r}=7.0$ but in case of air, that is, $P_{r}=0.71$, Nusselt number is positive at the lower plate and negative at the upper plate.
TABLE 4: Sherwood number.

\begin{tabular}{lccccc}
\hline Sl. no. & $t$ & $S_{c}$ & $K_{c}$ & $\mathrm{Sh}_{0}$ & $\mathrm{Sh}_{1}$ \\
\hline 1 & 0.4 & 100 & 2 & 5.9757644 & -0.612800788 \\
2 & 0.2 & 100 & 2 & 6.313751515 & 0.15838444 \\
3 & 0.4 & 617 & 2 & 7.026366229 & 0.470564281 \\
4 & 0.2 & 100 & 0 & 5.67128182 & -0.466307658 \\
\hline
\end{tabular}

Table 4 presents the variation of mass transfer for various values of $t, S_{c}$, and $K_{c}$. It is seen that an increase in chemical reaction parameter $K_{c}$ increases the mass transfer at the lower plate and decreases it at the other. Further, it is to note that Sherwood number at both the plates increases as $S_{c}$ increases, that is, for heavier species mass transfer increases at the plates. The effect of increase in time span is to reduce the rate of mass transfer at both plates.

Tables 5 and 6 show the effects of various parameters on mass flux both for unsteady and steady cases, respectively. From Table 5, it is observed that mass flux increases with the 
TABle 5: Mass flux (unsteady case).

\begin{tabular}{lccccccccc}
\hline Sl. no. & $t$ & $M$ & $S_{c}$ & $K_{c}$ & $N$ & $P_{r}$ & $D^{*}$ & $K_{p}$ \\
\hline 1 & 0.4 & 0.5 & 0.22 & 2 & 2 & 1.8 & 2 & 100 \\
2 & 0.2 & 0.5 & 0.22 & 2 & 2 & 1.8 & 2 & 100 \\
3 & 0.4 & 2 & 0.22 & 2 & 2 & 1.8 & 2 & 0.197836 \\
4 & 0.4 & 0.5 & 0.3 & 2 & 2 & 1.8 & 2 & 0.176572 \\
5 & 0.4 & 0.5 & 0.22 & 1 & 2 & 1.8 & 2 & 100 & 100 \\
6 & 0.4 & 0.5 & 0.22 & 2 & 4 & 1.8 & 2 & 0.057655 \\
7 & 0.4 & 0.5 & 0.22 & 2 & 2 & 2 & 2 & 0.568475 \\
8 & 0.4 & 0.5 & 0.22 & 2 & 2 & 1.8 & 4 & 100 & 0.091193 \\
9 & 0.4 & 0.5 & 0.22 & 2 & 2 & 1.8 & 2 & 0.166667 \\
\hline
\end{tabular}

TABle 6: Mass flux (steady case).

\begin{tabular}{lccccccc}
\hline Sl. no. & $M$ & $S_{c}$ & $K_{c}$ & $N$ & $D^{*}$ & $K_{p}$ \\
\hline 1 & 0.5 & 0.22 & 2 & 2 & 2 & 2 & 100 \\
2 & 2 & 0.22 & 2 & 2 & 2 & 100 & 0.119052 \\
3 & 0.5 & 0.3 & 2 & 2 & 2 & 100 & 0.104207 \\
4 & 0.5 & 0.22 & 1 & 2 & 2 & 100 & 0.119052 \\
5 & 0.5 & 0.22 & 2 & 4 & 4 & 100 & 0.195077 \\
6 & 0.5 & 0.22 & 2 & 2 & 2 & 0.122392 \\
7 & 0.5 & 0.22 & 2 & 2 & 0.5 & 0.094632 \\
\hline
\end{tabular}

increase in the values in $S_{c}, K_{c}, P_{r}$, and $D^{*}$ but the reverse effect is observed in case of $t, M, N$, and $K_{p}$ when the motion is unsteady. Hence, it is concluded that in case of unsteady motion more flux fluid was experienced with heavier species having low thermal diffusivity and increasing Dufour effect in the presence of constructive chemical reaction.

Now, if we analyse the steady case,we observe that more flux is measured with higher value of $N$ and $D^{*}$ whereas the reverse effect is observed in case of $M$ and $K_{p}$. In case of $S_{c}$ and $K_{c}$ there is no significant effect on mass flux.

Comparing both cases steady and unsteady motion, it is observed that an increase in $K_{c}$ and $D^{*}$ gives rise to higher flux whereas magnetic field yields less amount of flux.

\section{Conclusion}

(i) Under the influence of dominating mass diffusivity over thermal diffusivity with stronger Lorentz force the velocity is reduced at all points of the channel.

(ii) Low rate of thermal diffusion leads to increase the thickness of boundary layer.

(iii) Diffusion of heavier species leads to flow reversal. It is well marked in case of aqueous solution. Flow of aqueous solution in the presence of heavier species is prone to back flow.

(iv) Destructive reaction in the presence of heavier species leads to oscillatory flow.

(v) In case of steady state, no back flow occurs irrespective of high or low value of mass transfer coefficient and Dufour effects. (vi) Sharp rise and fall of temperature is the outcome of low diffusivity and high Dufour effects.

(vii) Dufour effect and chemical reaction rate in the presence of heavier species enhance the frictional drag.

(viii) Heat transfer bears same sign at both the plates in the presence of aqueous solution but in case of air it is of opposite sign.

(ix) Mass transfer increases at both plates due to the presence of heavier species but it reduces with increasing time span.

(x) In case of unsteady motion more flux of fluid is experienced due to heavier species with low thermal diffusivity. Dufour effect enhances the flux both in steady and unsteady motion.

\section{Appendices}

\section{A.}

The complementary error function is given by

$$
\begin{gathered}
\operatorname{erfc}(\mathrm{x})=\frac{2}{\sqrt{\pi}} \int_{x}^{\infty} e^{-\lambda^{2}} d \lambda, \\
f\left(x_{1}, x_{2}, x_{3}, x_{4}, x_{5}\right) \\
=\frac{1}{2} \exp \left(\frac{x_{4} x_{5}}{x_{3}}\right) \\
\times\left[\exp \left(-x_{2} \sqrt{\frac{x_{4}}{x_{3}}+x_{1}}\right)\right.
\end{gathered}
$$




$$
\begin{aligned}
& \times \operatorname{erfc}\left(\frac{x_{2}}{2 \sqrt{x_{5}}}-\sqrt{\left(\frac{x_{4}}{x_{3}}+x_{1}\right) x_{5}}\right) \\
& +\exp \left(x_{2} \sqrt{\frac{x_{4}}{x_{3}}+x_{1}}\right) \\
& \left.\times \operatorname{erfc}\left(\frac{x_{2}}{2 \sqrt{x_{5}}}+\sqrt{\left(\frac{x_{4}}{x_{3}}+x_{1}\right) x_{5}}\right)\right] \text {, } \\
& g\left(x_{1}, x_{2}, x_{3}, x_{4}, x_{5}\right) \\
& =\frac{1}{2} \sqrt{\frac{x_{4}}{x_{3}}+x_{1}} \exp \left(\frac{x_{4} x_{5}}{x_{3}}\right) \\
& \times\left[\exp \left(x_{2} \sqrt{\frac{x_{4}}{x_{3}}+x_{1}}\right)\right. \\
& \times \operatorname{erfc}\left(\frac{x_{2}}{2 \sqrt{x_{5}}}+\sqrt{\left(\frac{x_{4}}{x_{3}}+x_{1}\right) x_{5}}\right) \\
& -\exp \left(-x_{2} \sqrt{\frac{x_{4}}{x_{3}}+x_{1}}\right) \\
& \left.\times \operatorname{erfc}\left(\frac{x_{2}}{2 \sqrt{x_{5}}}-\sqrt{\left(\frac{x_{4}}{x_{3}}+x_{1}\right) x_{5}}\right)\right] \\
& -\frac{2}{\sqrt{\pi}} \exp \left(-\left(\frac{x_{3}^{2}}{4 x_{5}}+x_{1} x_{5}\right)\right) \text {. }
\end{aligned}
$$

B.

$$
\begin{aligned}
A_{1}= & -D^{*}\left[\frac{S_{c}}{P_{r}-S_{c}}+P_{r}-S_{c}\right], \\
A_{2}= & -\frac{1-S_{c}}{S_{c} K_{c}-M-\left(1 / K_{p}\right)} \\
& \times\left[N+\frac{S_{c} D^{*}\left(K_{c}-M-\left(1 / K_{p}\right)\right)}{S_{c} K_{c}\left(1-P_{r}\right)+\left(M+\left(1 / K_{p}\right)\right)\left(P_{r}-S_{c}\right)}\right], \\
A_{3}= & \frac{1-P_{r}}{M+\left(1 / K_{p}\right)} \\
& \times\left[1+\frac{S_{c} D^{*}\left(K_{c}-M-\left(1 / K_{p}\right)\right)}{S_{c} K_{c}\left(1-P_{r}\right)+\left(M+\left(1 / K_{p}\right)\right)\left(P_{r}-S_{c}\right)}\right], \\
A_{4}= & \frac{D^{*} P_{r}\left(P_{r}-S_{c}\right)}{S_{c} K_{c}\left(1-P_{r}\right)+\left(M+\left(1 / K_{p}\right)\right)\left(P_{r}-S_{c}\right)}, \\
A_{5}= & \frac{D^{*}-N}{S_{c} K_{c}-M-\left(1 / K_{p}\right)},
\end{aligned}
$$

$$
\begin{aligned}
& A_{6}=\frac{D^{*}+1}{M+\left(1 / K_{p}\right)}, \\
& A_{7}=\frac{K_{c}\left(D^{*}+1\right)-\left(M+\left(1 / K_{p}\right)\right)(N+1)}{\left(M+\left(1 / K_{p}\right)\right)\left(K_{c}-M-\left(1 / K_{p}\right)\right)}, \\
& A_{8}=\frac{D^{*}-N}{K_{c}-M-\left(1 / K_{p}\right)}, \\
& A_{9}=\frac{-S_{c} K_{c}\left(D^{*}+1\right)+\left(M+\left(1 / K_{p}\right)\right)(N+1)}{\left(M+\left(1 / K_{p}\right)\right)\left(S_{c} K_{c}-M-\left(1 / K_{p}\right)\right)} .
\end{aligned}
$$

\section{References}

[1] B. K. Jha and A. O. Ajibade, "Free convection heat and mass transfer flow in a vertical channel with the Dufour effect," Journal of Process Mechanical Engineering, vol. 224, no. 2, pp. 91-101, 2010.

[2] V. M. Soundalgekar and S. P. Akolkar, "Effects of free convection currents and mass transfer on flow past a vertical oscillating plate," Astrophysics and Space Science, vol. 89, no. 2, pp. 241-254, 1983.

[3] N. G. Kafoussias and E. W. Williams, "Thermal-diffusion and diffusion-thermo effects on mixed free-forced convective and mass transfer boundary layer flow with temperature dependent viscosity," International Journal of Engineering Science, vol. 33, no. 9, pp. 1369-1384, 1995.

[4] B. K. Jha and A. K. Singh, "Soret effects on free-convection and mass transfer flow in the stokes problem for a infinite vertical plate," Astrophysics and Space Science, vol. 173, no. 2, pp. 251255, 1990.

[5] N. G. Kafoussias, "MHD thermal-diffusion effects on free-convective and mass-transfer flow over an infinite vertical moving plate," Astrophysics and Space Science, vol. 192, no. 1, pp. 11-19, 1992.

[6] M. S. Alam, M. M. Rahman, and M. A. Samad, "Dufour and Soret effects on unsteady MHD free convection and mass transfer flow past a vertical porous plate in a porous medium," Nonlinear Analysis. Modelling and Control, vol. 11, no. 3, pp. 217226, 2006.

[7] M. S. Alam, M. M. Rahman, M. Ferdous, K. Maino, E. Mureithi, and A. Postelnicu, "Diffusion-thermo and thermal-diffusion effects on free convective heat and mass transfer flow in a porous medium with time dependent temperature and concentration," International Journal of Applied Engineering Research, vol. 2, no. 1, pp. 81-96, 2007.

[8] O. A. Beg, T. A. Beg, A. Y. Bakier, and V. R. Prasad, "Chemicallyreacting mixed convective heat and mass transfer along inclined and vertical plates with soret and Dufour effects: numerical solution," International Journal of Applied Mathematics and Mechanics, vol. 5, no. 2, pp. 39-57, 2009.

[9] Z. Dursunkaya and W. M. Worek, "Diffusion-thermo and thermal-diffusion effects in transient and steady natural convection from a vertical surface," International Journal of Heat and Mass Transfer, vol. 35, no. 8, pp. 2060-2065, 1992.

[10] C. R. A. Abreu, M. F. Alfradique, and A. S. Telles, "Boundary layer flows with Dufour and Soret effects: I: forced and natural convection," Chemical Engineering Science, vol. 61, no. 13, pp. 4282-4289, 2006. 
[11] E. Osalusi, J. Side, and R. Harris, "Thermal-diffusion and diffusion-thermo effects on combined heat and mass transfer of a steady MHD convective and slip flow due to a rotating disk with viscous dissipation and Ohmic heating," International Communications in Heat and Mass Transfer, vol. 35, no. 8, pp. 908-915, 2008.

[12] M. Anghel, H. S. Takhar, and I. Pop, "Dufour and Soret effects on free convection boundary layer over a vertical surface embedded in a porous medium," Studia Universitatis BabesBolyai. Matematica, vol. 11, no. 4, pp. 11-21, 2000.

[13] A. Postelnicu, "Influence of a magnetic field on heat and mass transfer by natural convection from vertical surfaces in porous media considering Soret and Dufour effects," International Journal of Heat and Mass Transfer, vol. 47, no. 6-7, pp. 1467-1472, 2004.

[14] B. Gebhart and L. Pera, "The nature of vertical natural convection flows resulting from the combined buoyancy effects of thermal and mass diffusion," International Journal of Heat and Mass Transfer, vol. 14, no. 12, pp. 2025-2050, 1971.

[15] U. N. Das, R. Deka, and V. M. Soundalgekar, "Effects of mass transfer on flow past an impulsively started infinite vertical plate with constant heat flux and chemical reaction," Forschung im Ingenieurwesen, vol. 60, no. 10, pp. 284-287, 1994.

[16] S. S. Saxena and G. K. Dubey, "Unsteady MHD heat and mass transfer free convection flow of a polar fluid past a vertical moving porous plate in a porous medium with heat generation and thermal diffusion," Advances in Applied Science Research, vol. 2, no. 4, pp. 259-278, 2011.

[17] K. Raveendra Babu, A. G. Vijaya Kumar, and S. V. K. Varma, "Diffusion-thermo and radiation effects on MHD free convective heat and mass transfer flow past an infinite vertical plate in the presence of a chemical reaction of first order," Advances in Applied Science Research, vol. 3, no. 4, pp. 2446-2462, 2012.

[18] K. Sudhakar, R. Srinibasa Raju, and M. Rangamma, "Chemical reaction effect on an unsteady MHD free convection flow past an infinite vertical accelerated plate with constant heat flux, thermal diffusion and diffusion thermo," International Journal of Modern Engineering Research, vol. 2, no. 5, pp. 3329-3339, 2012.

[19] L. Debanath and D. Bhatta, Integral Transforms and Their Applications, Applications of Laplace Transforms, Chapman and Hall/ CRC, Taylor and Francies Group, London, UK, 2007.

[20] K. R. Cramer and S. I. Pai, Magnetofluid Dynamics for Engineers and Applied Physics, McGraw-Hill, New York, NY, USA, 1973.

[21] I. Pop, "Effect of Hall current on hydromagnetic flow near an accelerated plate," International Journal of Physics and Mathematical Sciences, vol. 5, p. 375, 1971.

[22] M. A. Hossain and K. Mohammad, "Effect of hall current on hydromagnetic free convection flow near an accelerated porous plate," Japanese Journal of Applied Physics, vol. 27, no. 8, pp. 15311535, 1988.

[23] P. K. Rath, G. C. Dash, and A. K. Patra, "Effect of Hall current and chemical reaction on MHD flow along an exponentially accelerated porous flat plate with internal heat absorption/generation," Proceedings of the National Academy of Sciences India A, vol. 80, no. 4, pp. 295-308, 2010. 

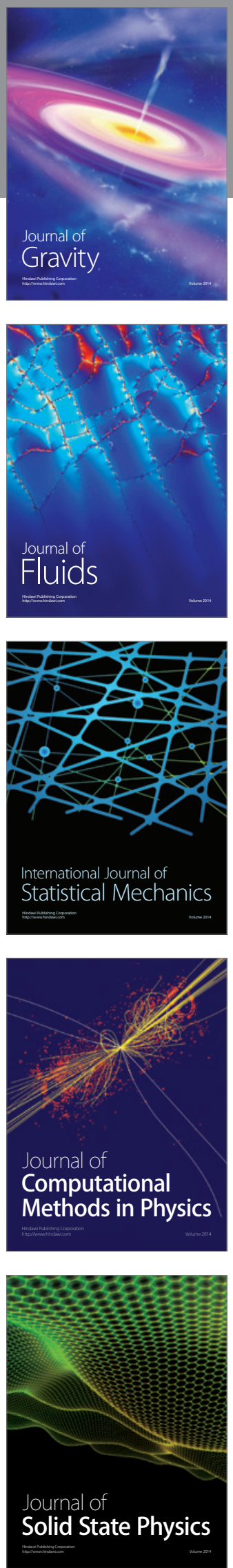

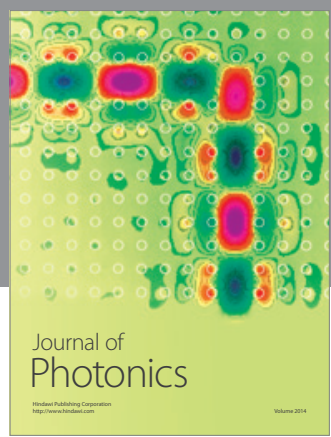

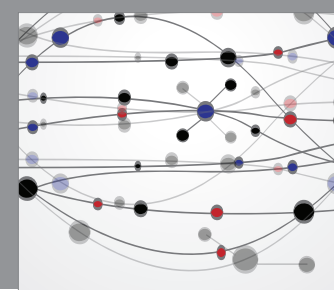

The Scientific World Journal

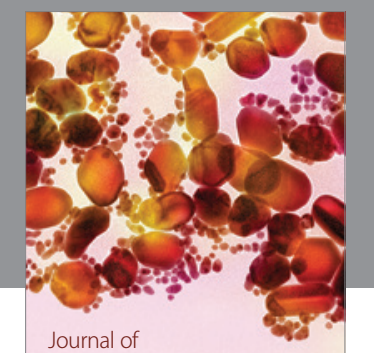

Soft Matter
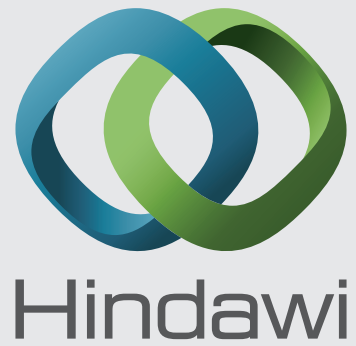

Submit your manuscripts at

http://www.hindawi.com
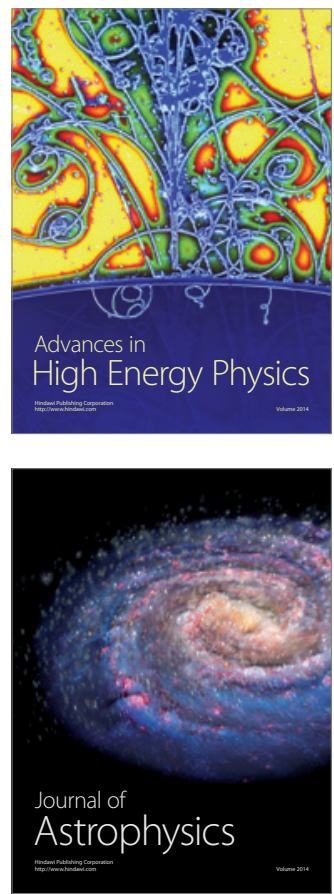
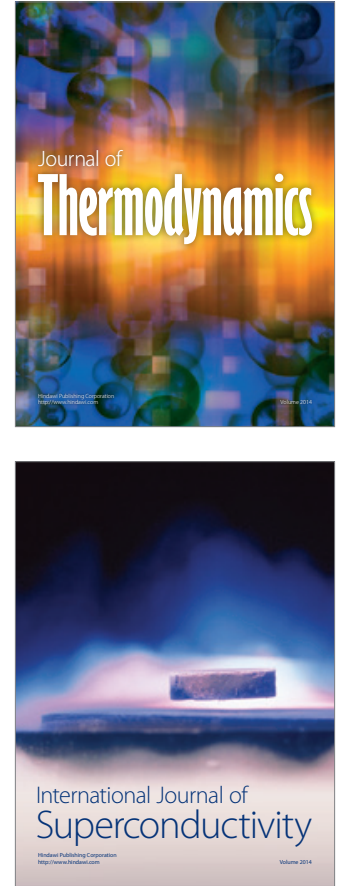
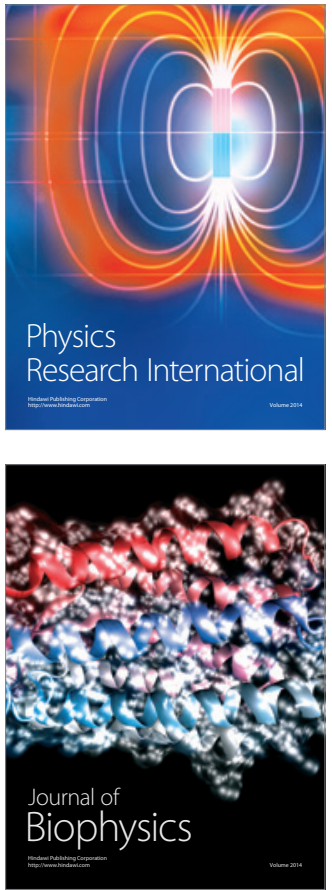
